NHGRI GWAS Catalog $(2005-2011)=5903$ Entries
4,789 Unique GWAS SNPs
342 Diseases/Traits
745 GWAS Publications

Filter by blood lipid related traits: LDL, HDL, TG, HDL-TG, total cholesterol, cholesterol, lipid metabolism, TG-BP, response to statin

$$
\begin{aligned}
& \text { Blood lipid-related traits = } 535 \text { Entries } \\
& 293 \text { Unique GWAS SNPs } \\
& 24 \text { GWAS publications }
\end{aligned}
$$

Remove studies based on non-

European/European American subjects

Studies with European/European American subjects $=490$ Entries 260 Unique GWAS SNPs

19 GWAS publications

140 independent GWAS signals (>100 kb apart)

Expand by including SNPs in

LD (HapMap CEU, $r^{2}>0.3$ )

\title{
6,230 SNPs
}

$\prod$ Search for enhancer-like epigenetic markings in the 500 bp region containing the SNP in HepG2 cells

\section{4 unique putative eSNP from 69 unique GWAS SNPs}

Filter by LD to select putative enhancer for experimental validation $\left(r^{2}>0.8\right.$ and $\left.D^{\prime}>0.9\right)$

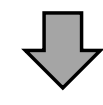

16 unique putative eSNPs from 36 GWAS SNPs

Remove 9 SNPs located within predicted promoters

7 putative eSNPs located in intronic, 5', 3' or intergenic regions that were cloned and tested in luciferase assay (HepG2 and Huh-7 cells)

\section{S1 Fig. From GWAS signals to liver-specific eSNPs.}

